\title{
Schwannoma de colon. Reporte de un caso.
}

\author{
Marlen Vega-Vega', Claudio Orlich-Carranza ${ }^{2}$, Hernando Valverde-Lozano ${ }^{3}$.
}

\begin{abstract}
Los schwannomas del tracto gastrointestinal son muy raros, los más comúnmente encontrados son los de estómago. Se presenta un caso de un schwannoma de colon, el cual es aún menos común. Los schwannomas se han asociado con neurofibromatosis. La incidencia es igual en ambos sexos y el rango de edad es amplio, desde los 18 hasta los 87 años. Los síntomas más hallados son el sangrado, el dolor abdominal y la obstrucción intestinal. El método diagnóstico más certero es la tomografía axial computarizada. El tratamiento es quirúrgico y consiste en la resección completa del tumor; la quimioterapia y radioterapia no han mostrado resultados favorables. El pronóstico depende principalmente del tamaño del tumor, edad mayor de siete años, la necrosis del $25 \%$ del tumor o más y la presencia de neurofibromatosis.
\end{abstract}

Descriptores: Schwannoma, colon, diagnóstico

Recibido: 31 de octubre, 2002

Aceptado: 18 de marzo, 2003

Los schwannomas, también conocidos como neurilemoma, neurinoma y fibroblastoma perineural; son tumores benignos de origen neurogénico. Difieren de los neurofibromas en que éstos consisten de una mezcla de células que se originan en la vaina nerviosa y los schwannomas exclusivamente de células de schwan.

La mayoría de los schwannomas son benignos, una pequeña parte de ellos se consideran malignos, y con potencial de metastatizar, estos son los conocidos como schwannomas malignos, neurofibrosarcomas, sarcomas neurogénicos o más correctamente, como tumores malignos de la vaina de nervios periféricos. La degeneración maligna de tumores benignos es rara. El diagnóstico diferencial desde el punto de vista patológico incluye fibrosarcoma, sarcoma sinovial y leiomiosarcomas.

\section{Reporte de caso}

Un hombre de 44 años, sin antecedentes personales patológicos ni heredo- familiares de importancia, consultó por un cuadro de 1 mes de evolución de estreñimiento y disminución del calibre de las heces, así como tenesmo, poliuria, astenia, adinamia, pérdida de aproximadamente cinco kilos de peso en el último mes, sensación de masa en hipogastrio y dolor en dicha zona, al examen físico se palpaba una masa ocupando

\section{Residente de Cirugía General \\ Jefe del Servicio \\ Asistente del Servicio}

Servicio de Cirugía General y Laparoscópica. Hospital San Juan de Dios

Correspondencia:

Marlen Vega Vega. E-mail: Marlen_Vega_Vega@yahoo.es todo el hipogastrio de consistencia dura y no móvil. Los exámenes de laboratorio mostraron una hemoglobina de 11.7 grs/dl, el hematocrito en $37 \%$ con anisocitosis, microcitosis, tipocromía, leucocitos, electrolitos, y las pruebas de función hepática estaban normales, la alfafetoproteínas estaban en 1.54 unidades internacionales por mililitro $(0.5-5.5)$, el antígeno carcinoembrionario en 2.56 nanogramos por mililitro (normal hasta 4.1); el antígeno prostático específico en 0.86 nanogramos por mililitro (hasta 4.0), y el CA 125 en 5.66 unidades internacionales por mililitro (hasta 21). Radiografía de tórax normal. El ultrasonido abdominal mostró un segmento intestinal de 15 a $20 \mathrm{cms}$, con pared gruesa, de 1 a $2 \mathrm{cms}$, formando una estructura sacular con gas y líquido intestinal en su interior, sin especificar segmento. La cistografía mostró una mega vejiga con desplazamiento anterior y hacia arriba relacionado con compresión extrínseca en su cara posterior, con contornos lisos bien delimitados mayor opacidad del medio en su parte superior. El colon por enema mostró en el recto sigmoides una zona disminuida de calibre de $13 \mathrm{cms}$ rígida, que no se distiende adecuadamente en relación con compresión por masa extrínseca, la mucosa se aprecia sin alteraciones (Figura 1).

En la cistoscopía se observó la presencia de una masa pélvica que desplaza la uretra posterior y la vejiga hacia delante. La tomografía axial computarizada evidenció una masa hipogástrica de la línea media, que midió en el diámetro transversal $12.6 \mathrm{cms}$ y en el anteroposterior, $8 \mathrm{cms}$, con una densidad mixta con tejido sólido, líquido y gas, que involucra además el ileon terminal, y se acompaña de líquido en el fondo de saco rectovesical (Figura 2 y 3 ).

Se realizó una laparotomía exploratoria, que reveló una masa pélvica que abarcaba el ciego e invadía el colon sigmoides, se 


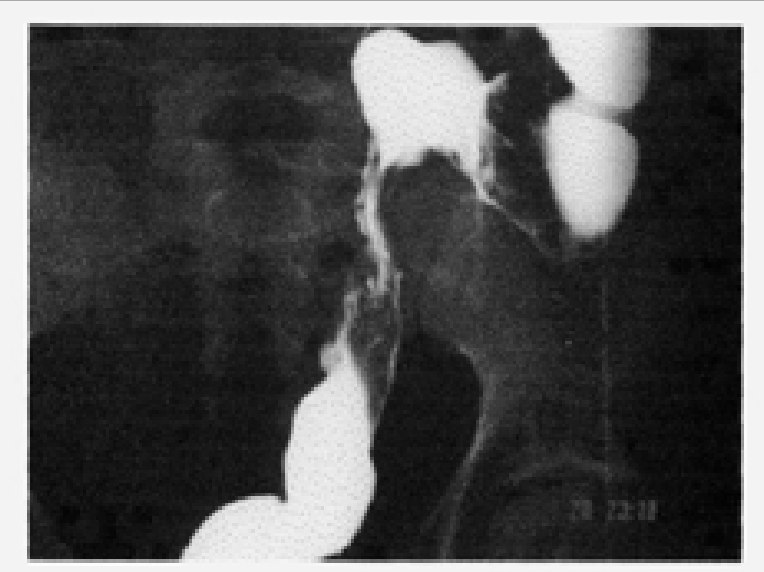

Figura 1. Colon por enema que muestra en recto sigmoides una zona disminuida de calibre por compresión extrínseca.

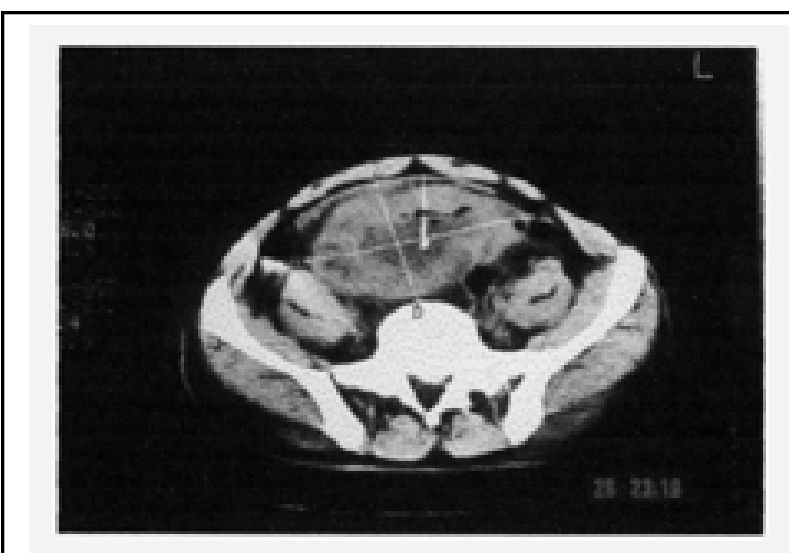

Figura 2.Tomografía Axial Computarizada de abdomen que muestra masa hipogástrica de $12.6 \mathrm{~cm}$ de diámetro antero posterior.

extiende además al retroperitoneo y al ileon terminal, de consistencia dura y aspecto al corte como de "carne de pescado". Fue imposible resecar la masa, por la gran invasión local.

\section{Hallazgos histopatológicos}

La biopsia corresponde a tejido fibrótico, eosinófilo, con abundante cantidad de células fusiformes, donde es posible identificar un patrón de crecimiento Antoni A, que corresponde a grupos de células alargadas con extensiones citoplasmáticas, con escasa matriz de estroma y Antoni B, donde se identifica un tejido celular menos denso; además se identificaron cuerpos de Verocay; dichos cambios histológicos fueron compatibles con un schwannoma.

\section{Discusión}

Los schwannomas surgen de las células de Schwann que envuelven los nervios periféricos y comprimen a un lado el nervio periférico residual; histológicamente son células fusiformes de la vaina nerviosa ${ }^{1}$.
Los schwannomas del tracto gastrointestinal son muy poco comunes. Lo son más en el estómago. Solo pocos casos se han documentado en el colon y recto, y son poco caracterizados.

Inicialmente son tumores benignos, que en raras ocasiones sufren malignización.

La incidencia es igual en hombres y en mujeres, en un rango de edad amplio, de 18 a 87 años con una media de edad de 65 años.

Cuando son de colon, la localización más común es en el ciego, seguido de recto sigmoides, luego el transverso, el colon descendente y, por último, el recto ${ }^{5}$. Se han reportado lesiones en todo el intestino grueso, las cuales son muy raras y el tratamiento es una proctocolectomía ${ }^{8}$.

Los tumores comúnmente se presentan como lesiones polipoideas intraluminales, a menudo con ulceración de la mucosa.

Los síntomas de presentación más comunes son el sangrado rectal, la obstrucción colónica y el dolor abdominal; también pueden manifestarse como masas palpables ${ }^{3}$.

Los hallazgos radiológicos no son específicos, pero ayudan a identificar y definir la extensión de la masa antes de la cirugía. Se debe realizar una Tomografía Axial Computarizada, con doble medio de contraste de tórax y abdomen.

Los schwannomas del tracto gastrointestinal se originan del plexo neural de Auerbach, de la subserosa, a lo largo del borde antimesentérico, y pueden ser intraluminales o intramurales.

Los pacientes con neurofibromatosis tienen la tendencia a desarrollar tumores neurogénicos en el tracto gastrointestinal, en aproximadamente un $11 \%{ }^{2}$; en el estómago es diez veces más común que en el intestino delgado; los schwannomas pancreáticos pueden ser benignos o malignos, los cambios

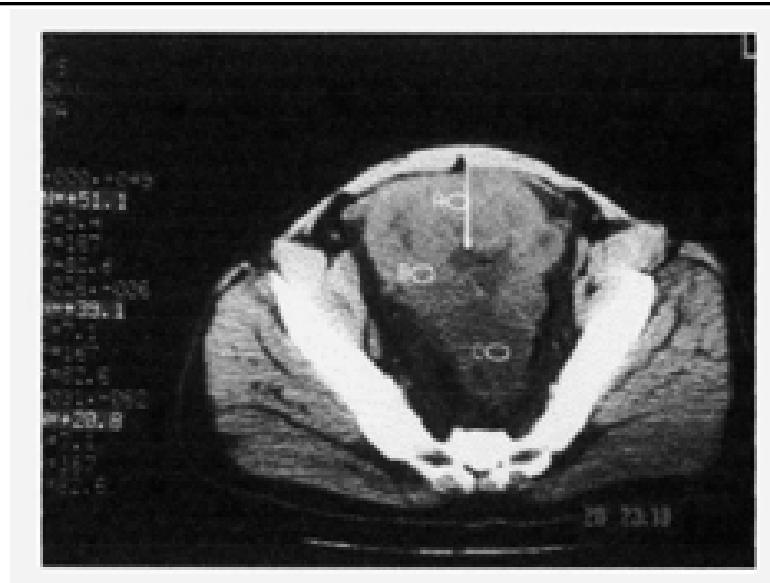

Figura 3. Tomografía Axial Computarizada de abdomen en corte pélvico que muestra masa con diferentes densidades que ocupa gran parte de la pelvis. 
quísticos del tumor se han asociado con neurofibromatosis de von Recklinghausen ${ }^{4}$.

Histológicamente tienen una cápsula de tejido conectivo que los envuelve con moderada densidad celular, con distribución irregular. La arquitectura celular permite individualizar los dos tipos de estructura. El tipo A es denso, con rasgo de fibras paralelas, con células arremolinadas o en empalizada (nódulos de Verocay) ${ }^{9}$. En el tipo B, la densidad celular es menor, se pueden encontrar microquistes que coalescen y forman quistes grandes. Estos dos tipos de presentación celular se pueden hallar en un mismo tipo de tumor. La forma $\mathrm{B}$ es la regresiva. Las variantes histológicas que se pueden identificar son tres; la más común es la de células en huso con patrón trabecular y cuerpos de Verocay o no, típicamente rodeados por células linfoides con centros germinales y atipia nuclear focal, se encuentra más comúnmente en el ciego y la mayoría se presentan como pólipos intraluminales con ulceración de la mucosa, causando sangrado rectal profuso. También se encuentra la variante epitelioide que usualmente se ubica en el colon sigmoide y el descendente, y la variable plexiforme está compuesta por múltiples nódulos de células de Schwann ${ }^{7}$.

Meis et $\mathrm{al}{ }^{6}$ revisaron 78 pacientes y el rango de sobrevida varió de 2 meses a 19 años, con una media de 45 meses, el 60\% de los pacientes tuvieron recurrencia local y en la mitad, a los 24 meses. Aproximadamente el 50\% desarrolló metástasis en 12 meses. Los sitios más comunes de metástasis son, en orden descendente de frecuencia: pulmón, nódulos linfáticos, hígado, hueso,tejidos blandos de cavidad abdominal,cerebro, riñón, páncreas, glándulas adrenales.

El peor pronóstico se asocia con gran tamaño, edad mayor de siete años, necrosis del tumor de un $25 \%$ o más y la presencia de neurofibromatosis.

El tratamiento es quirúrgico, alivia los síntomas, además es difícil excluir malignidad en una sola biopsia de material.

Hay pocas evidencias de que la quimioterapia y la radioterapia tengan algún efecto beneficioso adicional sobre la cirugía. El factor contribuyente más significativo para la sobrevida es la completa resección del tumor en la cirugía inicial.

\section{Abstract}

Schwannomas of the gastrointestinal tract are uncommon, gastric schwannomas are the most common. We report a case of a colonic schwannoma, wich are even less frecuent. They occur in association with neurofibromatosis. Schwannomas occur equally in men and women, in a wide age range. Haemorrhage, abdominal pain and bowel obstruction the most common presenting features. Computerized tomography confirms the diagnosis. Primary treatment is the complete surgical excision. Radiation therapy and chemotherapy do any have additional benefit over surgery. Size, age greater than 7 years, more than $25 \%$ of tumor necrosis and neurofibromatosis are the most important factors regarding in prognosis.

\section{Referencias}

1. Banks, P, Kraybill, W. Patología para el cirujano. México: McGrawHill Interamericana, 1998.

2. Bees NR,Dicks-Mireaux C,Kiely EM. Gastric malignant schwannoma in a child. Br J Radiol 1997; 70 (837):952-955.

3. Brady KA,Mc Carron,JP, Vaughan D, Javidian P. Benign schwannoma of the retroperitoneal space:case report. J of Urol 1993; 150:179-181.

4. Hsiao WC, Lin PW, Chang KC. Benign retroperitoneal schwannoma mimicking a pancreatic cystic tumor: case report and literature review. Hepatogastroenterology 1998; 45(24):2418- 2420.

5. Jacobson B, Hirsch M, Lee J, Van Dam J, Shoji B, Farraye F. Multiple asymptomatic plexiform schwannomas of the sigmoid colon:a case report and view. Gastrointestinal Endoscopy 2001; 53 (7).

6. Meis JM,Enzinger FM, Martz KL, Neal JA. Malignant peripheral nerve sheath tumors (malignant schwannomas) in children. Am J Surg Pathol 1992; 16:694-707.

7. Miettinen M.,Shekitka M,Sobin L. Schwannomas in the colon an rectum. Am J Surg Pathol 2001; 25 (7): 846-855.

8. Nabeya Y, Watanabe Y, Tohnosu N, Yamazaki M,et al. Diffuse schwannoma involving the entire large bowel with huge extramural development: report of a case. Surg Today 1999; 29 (7): 637-641.

9. White W, Shiu MH,Rosenblun,MK,et al. Cellular Schwannoma. Cancer 1990; 66:1266-1275. 